\title{
Treatment of Supratentorial Spontaneous Intracerebral Hemorrhage Using Image-Guided Minimally Invasive Surgery: Initial Experiences of a Flat Detector CT-Based Puncture Planning and Navigation System in the Angiographic Suite
}

\author{
Z. Yang, B. Hong, Z. Jia, J. Chen, J. Ge, J. Han, J. Beilner, Y. Zhang, Y. Fang, and J. Liu
}

\begin{abstract}
BACKGROUND AND PURPOSE: The intracerebral hemorrhage drainage through minimally invasive approach is emerging as an alternative for traditional craniotomy, due to its improved survival rate and reduced complication rate. In this study, we investigated the feasibility and safety of a flat detector CT-based puncture planning and navigation system for minimally invasive hematoma drainage on patients with intracerebral hemorrhage.
\end{abstract}

MATERIALS AND METHODS: The minimally invasive hematoma drainage was performed on 21 hypertensive patients with intracerebral hemorrhage in the angiographic suite with the guidance of a flat detector CT-based puncture planning and navigation system. This system is integrated in the angiographic machine, and was used for 1) planning the needle path based on a preprocedural flat detector CT scan, 2) advancing the catheter with real-time fluoroscopic guidance, and 3) confirming the procedure outcome based on an immediate postprocedural flat detector CT. The surgery efficiency, accuracy, and the treatment outcome were measured and compared with the published data.

RESULTS: All procedures were successfully completed with the catheter placed $4 \pm 1 \mathrm{~mm}$ from the planned position. The average surgery time was $40 \pm 7$ minutes. The volume of the hematoma was reduced to $28 \pm 4 \%$ of the original volume. The Glasgow Coma Scale score was significantly improved from $10 \pm 1$ at the admission to $14 \pm 1$ at the discharge. The Extended Glasgow Coma Scale score also improved from $5 \pm$ 1 at the discharge to $6 \pm 1$ at the 6-month follow-up. No major complication, rebleeding, and mortality were observed in this study.

CONCLUSIONS: This flat detector CT-based needle guidance system provided a feasible, convenient, and safe way to perform the puncture and drainage of brain hematoma in the angiographic suite.

ABBREVIATIONS: $\mathrm{FDCT}=$ flat detector $\mathrm{CT} ; \mathrm{ICH}=$ intracerebral hemorrhage; $\mathrm{GCS}=$ Glasgow Coma Scale; $\mathrm{GOSE}=$ Extended Glasgow Coma Scale

S pontaneous intracerebral hemorrhage ( $\mathrm{ICH}$ ) affects more than 2 million people each year around the world. ${ }^{1}$ Timely medical intervention is critical for $\mathrm{ICH}$, as it is a medical emergency with a mortality rate range from $35 \%$ to $52 \%$ within 1 month. ${ }^{2}$ However, the current treatments for patients with ICH continue to be controversial, with several large-scale randomized trials reporting no significant benefit of early surgery, primarily craniotomy, over conservative treatment. ${ }^{3-5}$ In recent years, the surgical removal of hematoma through burr-holes is emerging as a minimally invasive alternative for conventional craniotomy because of its improved complication rate and survival rate. ${ }^{6}$ These new techniques usually involve stereo-

Received January 27, 2014; accepted after revision April 1.

From the Department of Neurosurgery (Z.Y., B.H., Z.J., J.C., Y.Z., Y.F., J.L.), Changhai Hospital, Second Military Medical University, Shanghai, China; and Siemens Ltd. China (J.G., J.H., J.B.), Healthcare Sector, Angiography \& Interventional X-Ray Systems, Shanghai, China.

Z. Yang and B. Hong contributed equally to this work as co-first authors.

Please address correspondence to Jianmin Liu, MD, Department of Neurosurgery, 168 Changhai Rd, Shanghai 200433, China; e-mail: renzhhuang@hotmail.com

http://dx.doi.org/10.3174/ajnr.A4009 tactic positioning and intraprocedural image guidance, such as endoscopic, traditional CT, intraoperative CT, and sonography-guided aspiration. ${ }^{6-8}$ These techniques have not yet been widely used because of expensive clinical costs such as the demand for specialized equipment, difficulties scheduling procedures on the diagnostic machines (eg, CT scan suite), and a lack of confirmed clinical benefit from large-scale clinical trials. Until now, the implementation of minimally invasive surgery for treating intracranial hemorrhage remains investigational, with only 2 ongoing large-scale randomized trials (Minimally Invasive Surgery and Thrombolytic Evacuation in ICH [MISTIE] and Clot Lysis Evaluating Accelerated Resolution III [CLEAR III]). ${ }^{9,10}$ MISTIE and CLEAR III are currently phase III trials aimed at comparing minimally invasive surgery combined with a thrombolysis agent with the best critical management alone for treating $\mathrm{ICH}$, and with minimally invasive surgery combined with placebo for treating intraventricular hemorrhage, respectively. Both of their early results indicated that the catheter location is one of the most important criteria for good surgery outcome, and careful planning and accurate execution is necessary for optimal catheter placement. $^{10}$ 
In this study, we attempted an affordable clinical solution for image-guided hematoma drainage that could be performed in the traditional angiographic suite without the need for specialized equipment. We implemented an innovative puncture planning and navigation system that is integrated into the angiographic machine for hematoma evacuation. This guided evacuation procedure based on an intraprocedural flat detector CT (FDCT), in which a 3D CT-like image was reconstructed from a rotational scan ${ }^{11}$ using a $\mathrm{C}$-arm angiographic machine. Commercial software (syngo iGuide needle guidance; Siemens, Erlangen, Germany) then facilitates the needle path planning that allows physicians to choose optimum entry and target points of the puncture, and automatically overlays the path onto the fluoroscopic images during the procedure for real-time guidance.

The feasibilities of this puncture planning and guidance system had been previously demonstrated in selective cervical nerve root block, ${ }^{12}$ percutaneous kidney puncture, ${ }^{13}$ and hepatic tumor ablation ${ }^{13}$ based on ex vivo animal models and real patients. Its application in the brain was only recently demonstrated on 6 patients with cerebrovascular ischemia for external ventricular drainage. ${ }^{14}$ In this study, we further expanded this technique on a more complicated brain procedure, intracranial hemorrhage evacuation in 21 hypertensive patients with ICH. Unlike the external ventricular drainage, the location of hemorrhage in pa-

Table 1: The GCS score of all 21 patients measured at admission and discharge

\begin{tabular}{ccccc}
\hline GCS Score & $\mathbf{7 - 9}$ & $10-12$ & $13-15$ & Average \\
\hline At admission (no. of patients) & $11(52 \%)$ & $10(48 \%)$ & 0 & $9.5 \pm 0.9$ \\
At discharge (no. of patients) & 0 & 0 & $21(100 \%)$ & $14.1 \pm 0.6$ \\
\hline
\end{tabular}

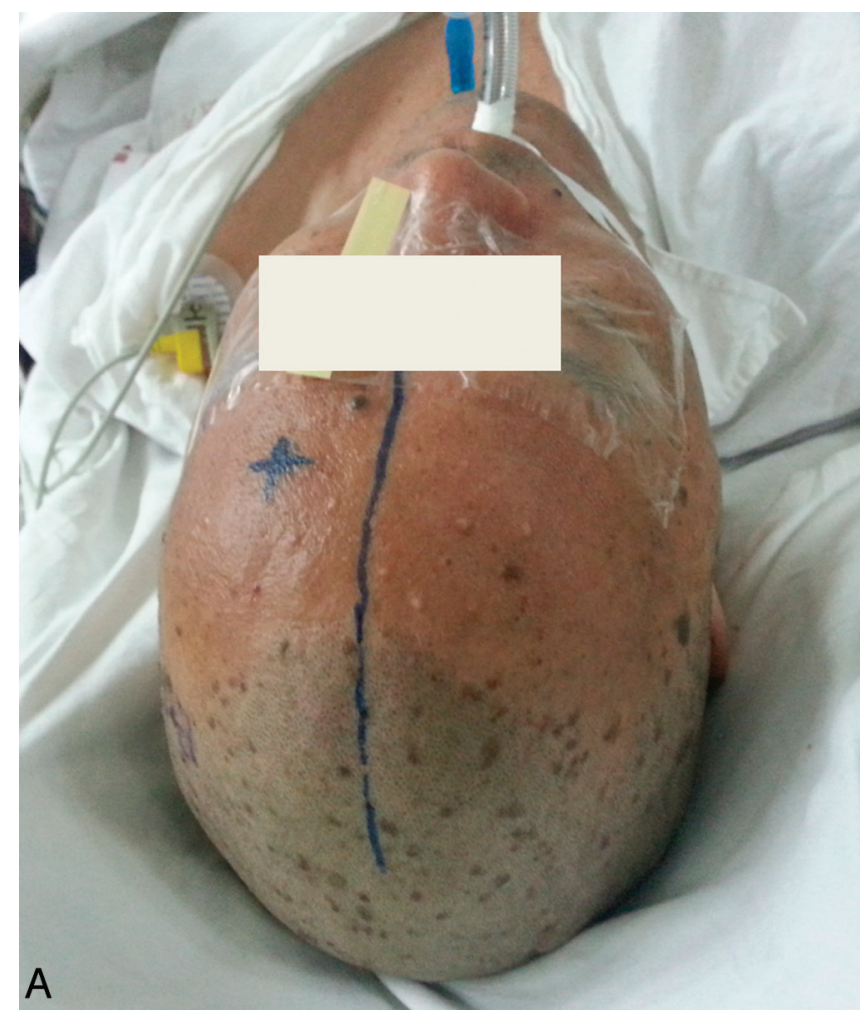

tients with ICH could vary from patient to patient, and could not be performed freehand without any image guidance. Moreover, we assessed the feasibility and the safety of this technique in terms of procedure length, puncture accuracy, drainage volume, and treatment outcome at the discharge and 6-month follow-up. This is also the first attempt to evaluate the short-term treatment outcome of FDCT-guided hemorrhage drainage using Glasgow Coma Scale (GCS) and Extended Glasgow Coma Scale (GOSE) scores.

\section{MATERIALS AND METHODS \\ Patients}

Between February 2011 and May 2011, 21 consecutive supratentorial hypertensive patients with ICH (15 men and 6 women, age 49 to 80 years, mean $59.3 \pm 7.9$ years) were included in this study. The inclusion criteria were 1) definite hematoma associated neurologic symptoms and signs; 2) no signs of brain herniation and acute rising intracranial pressure; 3 ) hematoma was deeply located, craniotomy operation of hematoma evacuation was difficult, or might cause more iatrogenic injury; 4) the hemorrhagic vascular diseases such as aneurysms or vascular malformations were definitely treated; and 5) informed patient consent. All patients had a history of hypertension. On admission, GCS score of the cohort was $9.5 \pm 0.9$ with details listed in Table 1 . Among them, 13 patients experienced various degrees of aphasia and 16 patients had contralateral limb muscle strength grades worse than 3. CT examination identified that 6 cases had hematoma in the left putamen, 10 cases in the right putamen, and 5 cases in the thalamus. There were also 2 patients whose hematoma had spread into
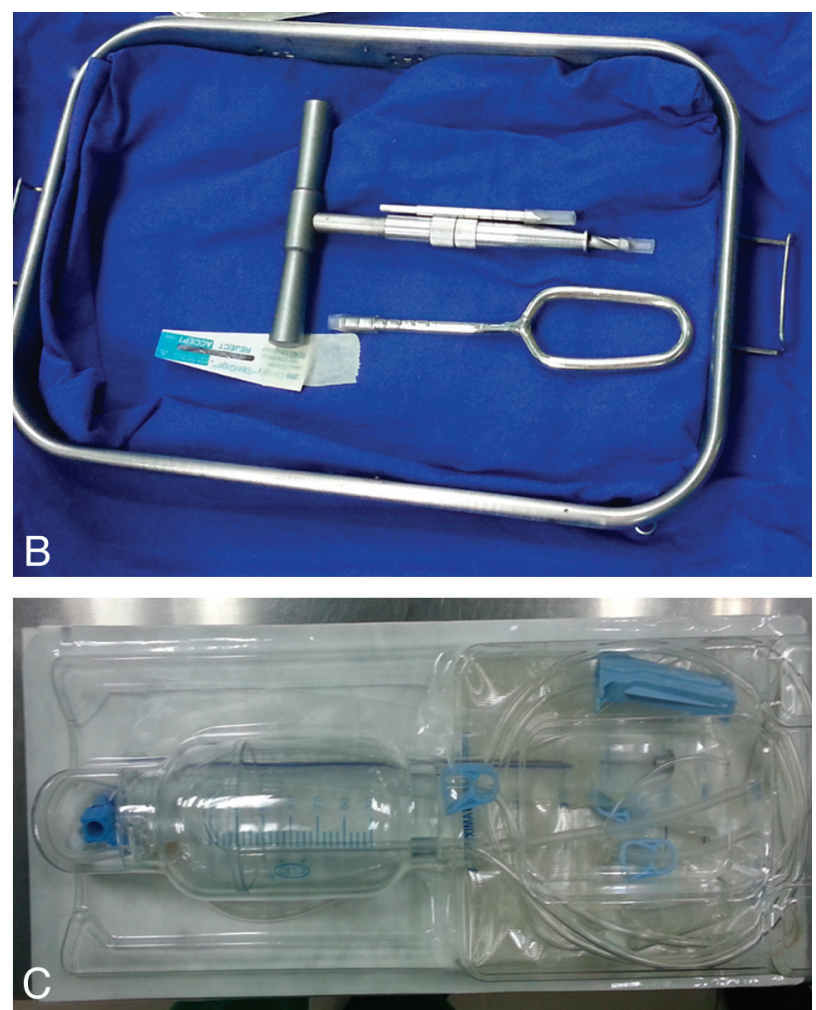

FIG 1. The generation of burr-hole for hematoma drainage. $A$, The burr-hole position is preselected by the surgeon before the puncture planning procedures, and marked on the patient's forehead as the blue cross. The surgeon then uses the hand drill, $B$, to manually drill a hole at the selected position. The drainage catheter, $C$, is then inserted into the burr-hole with the FDCT-based real-time guidance. 




FIG 2. The selection of the entry point and the target point is based on 3D FDCT images acquired immediately before the surgery in the angiographic suite. The planned target point is placed within the hematoma based on the preference of the surgeon. Its position is indicated by the center of the cross hair in sagittal ( $A$ ), coronal (B), and axial (C) axes. In our practice, the burr-hole is generated before the presurgery FDCT, hence we could directly place the entry point at the center of the burr-hole (small green cross in D).

the ventricle. The time spans from the onset of the hemorrhage to surgery ranged from 12 hours to 7 days, with an average of $3.5 \pm$ 1.5 days.

\section{Anesthesia}

Fifteen patients were under local anesthesia with certain degrees of sedation, the other 6 patients who were too dysphoric to be cooperative were under general anesthesia.

\section{Needle Planning and Path Guidance}

All procedures were performed in an angiographic suite that conformed to ISO 7 (Class of 10,000) standards with average of air flow velocity of 10-15 feet/minute. During the procedure, all patients lay on the angiographic table in supine position. The puncture was generally performed at the location of $3 \mathrm{~cm}$ superior to the supraorbital ridge and $2.5 \mathrm{~cm}$ lateral to midline using a manual twist drill (Fig 1). After the establishment of a burr-hole, 1 FDCT acquisition was performed on a C-arm angiographic system (Artis zee Biplane, Siemens) with the following parameters: 20 -second acquisition protocol, $0.4^{\circ}$ increment, $512 \times 512 \mathrm{ma}-$ trix, $200^{\circ}$ total angle, and 496 projections. The cross-sectional images were then reconstructed on a commercially available workstation (syngo X-Workplace; Siemens) with section thickness of $5 \mathrm{~mm}$. After identification of the hemorrhage in the reconstructed image, the integrated puncture planning and guidance system (syngo iGuide needle guidance) was initiated. The syngo iGuide enabled preprocedural needle path planning, intraprocedural live guidance during needle progress, and postprocedural verification. During the needle path planning, the entry point and target point could be chosen based on reconstructed 3D FDCT images. In our cases, we slightly modified the workflow by generating the burr-hole before the acquisition of FDCT, hence the entry point was chosen by identifying the center of the burr-hole in the 3D FDCT scan (Fig $2 D$ ). The target point was chosen by selecting a point within the hemorrhage and $1 \mathrm{~cm}$ away from the posterior boundary of the hemorrhage (Fig 2A$C)$. The needle guidance software then automatically calculates the puncture depth and corresponding C-arm working angles in bull's eye view (Fig $3 A,-B$ ) and 2 progression views (Fig $3 C,-D$ ). After manual confirmation of the puncture path, we started the image-guided puncture by overlaying the planned path onto the real-time fluoroscopic images. After the puncture and the initial drainage, a postprocedural FDCT was performed immediately to confirm the location of the drainage tube.

\section{Postprocedural Patient Care}

The liquefied hematoma was partially drained out immediately after the successful placement of the drainage tube. The disposable drainage system (Gred; ShanDong Gredmedic, Dalian, China) used in the procedure has a built-in U-shape tube that could prevent reversed flow of hematoma from drainage bag back to patient (as shown in Fig 1C). For 5 patients with large hematoma volume, a $20-\mathrm{mL}$ syringe with 1 to $2 \mathrm{~mL}$ negative pressure was used for gentle suction. Starting from the second day after the surgery, all patients received diluted saline urokinase ( 2 million units in $2 \mathrm{~mL}$ ) treatment 1 time per day. Dehydration was performed according to the results of intracranial pressure monitor.

\section{Surgery Evaluation}

The feasibility and safety of this image-guided hematoma drainage was evaluated based on the surgery efficiency and accuracy, and the treatment outcome. The efficiency of the surgery was represented by the total surgery time excluding anesthesia time, and the number of puncture attempts per surgery. The accuracy of the surgery was represented by the deviation between the planned and actual location of the needle tip. This distance was manually measured on the postprocedural FDCT as it has an automatic overlay of the planned puncture path.

The immediate treatment outcome was evaluated based on the hematoma drainage volume and the GCS score at the time of admission and discharge. The short-term outcome was evaluated based on the GOSE score at the time of the discharge and 6-month 

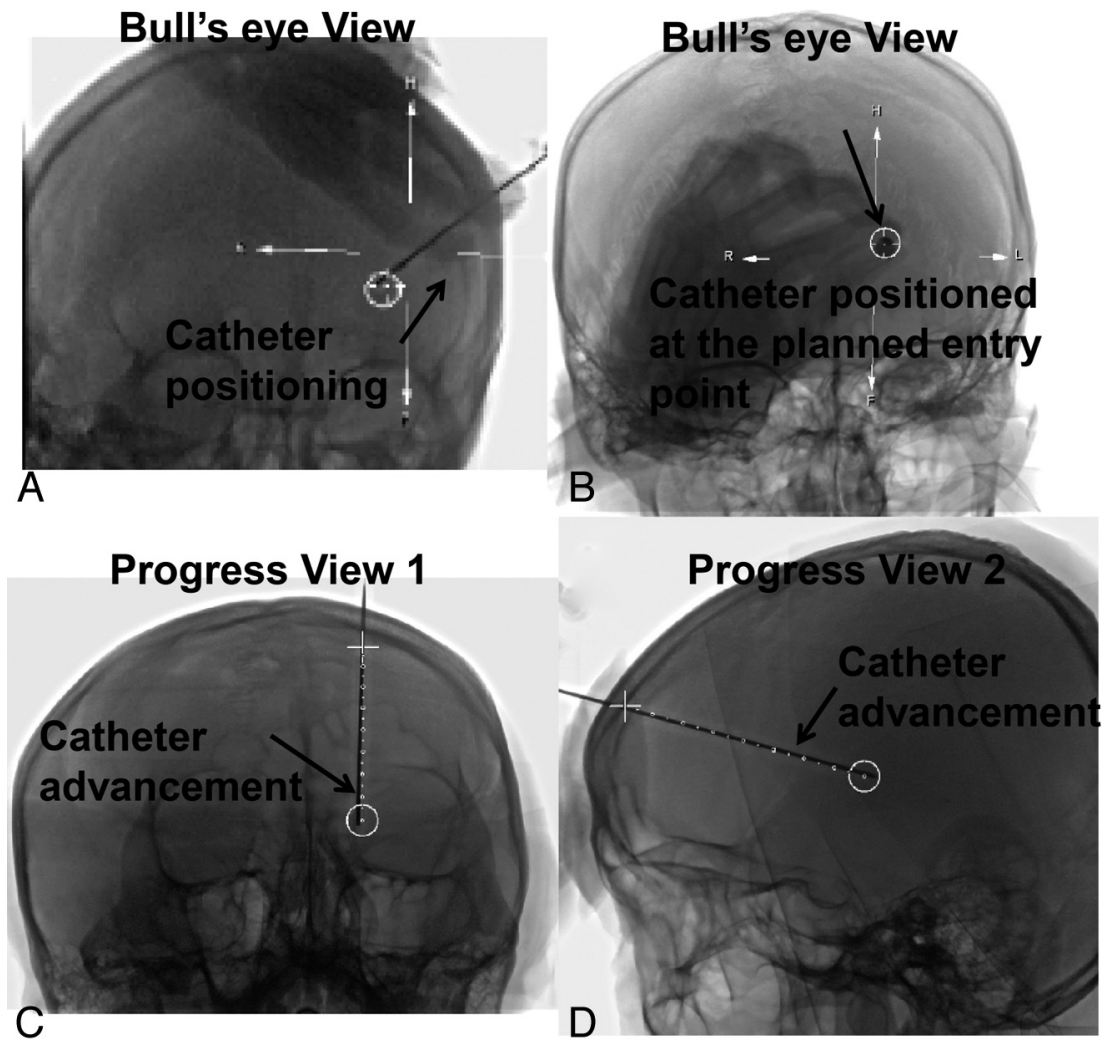

FIG 3. The catheter placement is guided by real-time fluoroscopic images with overlaid planned needle path (white dotted line in $A-D$ ). Under the bull's eye view ( $A$ and $B$ ), the surgeon adjusts the direction of the catheter until the catheter is in-line with the beam angle, and its tip overlaps with the planned entry point (white circle) ( $C$ and $D$ ). The surgeon then slowly advances the catheter while watching the real-time fluoroscopy acquired perpendicular to the planned needle path.

follow-up. The volumes of the hematoma were measured based on presurgery and postsurgery CT scan using the Tada formula.

\section{Statistical Analysis}

The results were analyzed with SPSS 13.0 edition (IBM, Armonk, New York). A $t$ test was performed to compare 1) the change of hematoma volume pre- and postsurgery, 2) the GCS score at the time of admission and discharge, and 3) the GOSE score at the time of discharge and 6-month follow-up.

\section{RESULTS}

The image-guided operation time ranged from 25 to 55 minutes, with mean length of $40 \pm 7$ minutes. All punctures succeeded in 1 attempt. The deviation between planned and actual needle point location ranged from 2 to $7 \mathrm{~mm}$, with mean deviation of $4 \pm 1$ $\mathrm{mm}$. The average drainage volume was $24 \pm 7 \mathrm{~mL}$, with average hematoma volume of $33 \pm 9 \mathrm{~mL}$ at the admission, and $9 \pm 2 \mathrm{~mL}$ at the discharge. The volume of hematoma was significantly reduced $(P<.001)$ and the drainage ratio ranged from $67 \%$ to $80 \%$, and averaged at $72 \pm 4 \%$. An exemplary patient hematoma shrinkage after this image-guided surgery was clearly shown on a series of CT scans in Fig 4. The GCS score was significantly improved from $10 \pm 1$ at the admission to $14 \pm 1$ at the discharge $(P<.001)$, with the details of the GCS score listed in Table 1 . The GOSE score also significantly improved from $5 \pm 1$ at the discharge to $6 \pm 1$ at the 6 -month follow-up $(P<.001)$, as listed in Table 2. All patients survived the procedure without major com- plications; however, 1 patient suffered from pneumonia due to nosocomial infection. Postoperative hospital stay was 5-8 days. Among 13 cases of aphasia, 3 patients could speak simple words, and 4 could voice. Among 16 cases of hemiplegia, 12 patients improved their muscle strength more than 1 level.

\section{DISCUSSION}

The prolonged operation time is one of the limitations for removing clot using craniotomy. In our experience with this FDCT-based puncture planning and navigation method, we were able to complete the procedure in around 40 minutes. This procedure time was significantly shorter than that of the conventional craniotomy treatment, which usually takes around 3 hours in our institute. It was also slightly faster than other reported image-guided surgery times, such as 89 minutes using intraoperative $\mathrm{CT}^{14}$ and 85 minutes using endoscopic navigation. ${ }^{7}$ The shortened workflow was mainly a benefit from the convenience of performing intraprocedural FDCT within the angiographic suite. FDCT, in general, has lower resolution than that of volumetric CT, but it is sufficient for the application of intracranial hematoma evacuation. First, the attenuation difference of the skull, the hemorrhage, and the normal brain tissue within the brain is large enough to be easily differentiated in a reconstructed 3D FDCT image (as shown in Fig 2). Second, the respiratory motion and the cardiac rhythm only minimally affect the brain tissue; hence its immobility could further enhance the image quality.

The key factor for hemorrhage evacuation through burr-holes is the precise positioning of the drainage catheter. The maximum deviation between planned and actual position of the drainage catheter was found to be less than $7 \mathrm{~mm}$ in our study. Moreover, postprocedural FDCT indicated that the catheter tips arrived at the center of the hemorrhage in all cases. The accurate placement of the catheter in our study was mainly due to the real-time guidance of the fluoroscopic image with overlaid planned needle path. The surgeon could make a slight adjustment to the trajectory path simultaneously as the catheter advanced to the planned location while watching the real-time fluoroscopy with overlaid planning needle path. By doing this, we could avoid the multiple punctures that tend to have a much higher chance of brain tissue damage than a single attempt. We also noticed that the small deviation still existed in some cases, and that could be due to the lack of bilateral guidance during catheter advancement. This should be taken into consideration in the future version of the needle guidance tool.

Theoretically, the accurate placement of the evacuation catheter could lead to better treatment outcome. The treatment outcome evaluation based on our patients' condition at discharge 


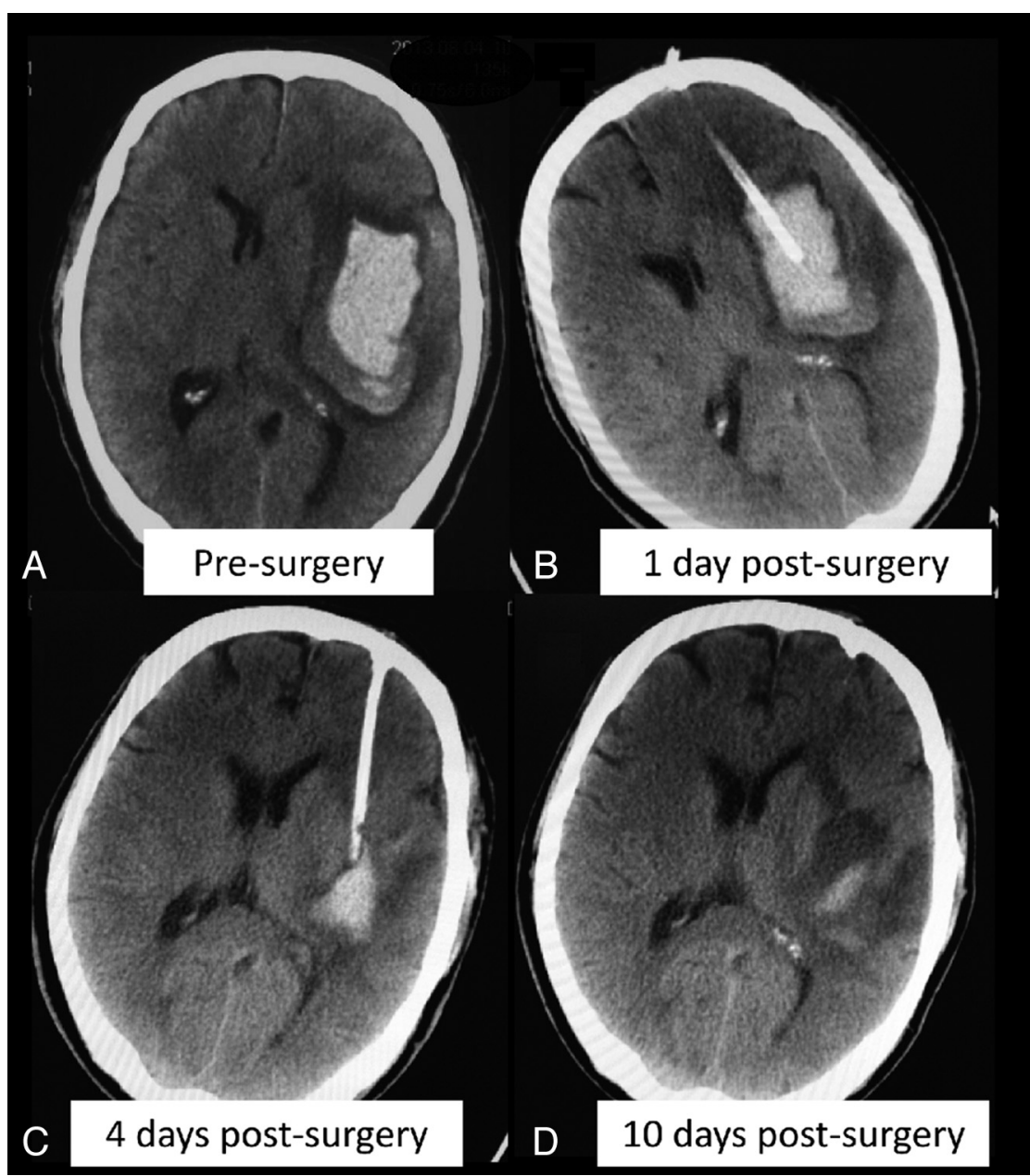

FIG 4. An exemplary patient case that was treated using this image-guided hemorrhage drainage procedure. The significantly reduced hematoma volume could be observed in a series of pre- and postsurgery CT scans.

Table 2: The GOSE score of all 21 patients measured at discharge and 6-month check-up

\begin{tabular}{lcccccc}
\hline \multicolumn{1}{c}{ GOSE Score } & $\mathbf{3}$ & $\mathbf{4}$ & $\mathbf{5}$ & $\mathbf{6}$ & $\mathbf{7}$ & Average \\
\hline At discharge (no. of patients) & $2(10 \%)$ & $7(33 \%)$ & $10(48 \%)$ & $1(5 \%)$ & $1(5 \%)$ & $4.6 \pm 0.9$ \\
At 6-month check-up (no. of patients) & 0 & $1(5 \%)$ & $8(38 \%)$ & $8(38 \%)$ & $4(19 \%)$ & $5.7 \pm 0.6$ \\
\hline
\end{tabular}

human head. In this way, the impact of the twist drill on the scalp and head shift will be excluded from the puncture planning; 3) Surgery timing: At least 24 hours after the onset of the hemorrhage. Although there were extensive reports of the benefit of ultra-early and early removal of the hematoma in the literature, the surgery timing is still controversial due to a lack of evidence-based support. In our opinion, the hematoma drainage could be easier and the risk of rebleeding could be lower when we allow at least 1 day for hematoma to liquefy.

One of the important benefits of this demonstrated FDCT/fluoro-assisted minimally invasive surgery was the low cost of the procedure, as it can be performed in the traditional angiographic suite, without adding extra cost to patients for specialized devices. The shortened operation time compared with craniotomy also significantly reduced the clinical cost of this procedure. This is especially useful for underdeveloped countries that experience high patient volume but have limited medical resources. In this way, more low-income patients will have the chance to receive timely treatment. Moreover, given the low cost and high performance of the procedure, this could potentially encourage more eligible patients to be recruited into a future prospective study.

One limitation of this study is that only patients with indications for surgery were included in this retrospective study. Although we attempted to compare the treatment outcome with published meta-

and 6-month follow-up supported this assumption. In our study, no patient showed recurrent bleeding or technique-related postsurgery complication, whereas the reported studies have shown that the complication rate of the stereotactic aspiration treatment was $17.9 \%-37.3 \%$, and the rebleeding incidence was $5 \%-13.3 \% .^{6,15-19}$

For the FDCT/fluoro-assisted hematoma evacuation in the angiographic suite, our experiences in 21 hypertensive patients with ICH were concluded as follows: 1) Puncture site selection: The frontal approach was preferred over the temporal approach because of the consideration that there is relatively less neurologic and vascular traffic in the frontal lobe area. In addition, puncturing and advancing the catheter from the forehead was convenient for operators because the bull's eye view and progression views in the $\mathrm{C}$-arm were nearly vertical and horizontal in direction, with minimal interference on operations and anesthesia. 2) Operation procedures: We suggested generating the burr-hole before the acquisition of planning FDCT for this specific application on a analysis results from large-scale clinical trials, the comparison was not based on the same patient baseline. Retrospectively matching the surgery group with patients receiving medical treatment only, however, could bring uncertainties such as different medical treatment strategies and varied treatment timings into comparison because patient treatments were originally conducted without clear guidelines. Hence, though the feasibility of this FDCT/fluoro-guided ICH evacuation was demonstrated, one of our immediate future works is to initiate a multicenter, prospective trial that compares the immediate and long-term treatment outcome of combined FDCT/fluoro-assisted minimally invasive surgery and urokinase treatment with conventional medical therapy only for patients with $\mathrm{ICH}$.

\section{CONCLUSIONS}

We demonstrated the feasibility of performing intracranial hemorrhage evacuation in an angiographic suite, with the assistance of puncture planning and guidance software that uses intraprocedural FDCT and fluoroscopy. According to our clinical outcomes, 
intraprocedural FDCT and integrated needle guidance software provided a feasible, convenient, and safe solution for hemorrhage evacuation. In the future, the benefit of this demonstrated imageguided ICH evacuation should be further investigated in a largescale prospective and randomized trial.

Disclosures: Jiajia Ge is employed by Siemens Healthcare. Siemens Healthcare supports some of the scientific activities on which this manuscript is based. Jingfeng Han is employed by Siemens Limited China. Janina Beilner is working for Siemens Limited China; Siemens equipment was used for this study.

\section{REFERENCES}

1. Qureshi AI, Mendelow AD, Hanley DF. Intracerebral haemorrhage. Lancet 2009;373:1632-44

2. Broderick J, Connolly S, Feldmann E, et al. Guidelines for the management of spontaneous intracerebral hemorrhage in adults: 2007 update: a guideline from the American Heart Association/American Stroke Association Stroke Council, High Blood Pressure Research Council, and the Quality of Care and Outcomes in Research Interdisciplinary Working Group. Stroke 2007;38:2001-23

3. Elliott J, Smith M. The acute management of intracerebral hemorrhage: a clinical review. Anesth Analg 2010;110:1419-27

4. Mendelow AD, Gregson BA, Fernandes HM, et al. Early surgery versus initial conservative treatment in patients with spontaneous supratentorial intracerebral haematomas in the International Surgical Trial in Intracerebral Haemorrhage (STICH): a randomised trial. Lancet 2005;365:387-97

5. Sykora M, Diedler J, Juttler E, et al. Intensive care management of acute stroke: surgical treatment. Int J Stroke 2010;5:170-77

6. Zhou H, Zhang Y, Liu L, et al. A prospective controlled study: minimally invasive stereotactic puncture therapy versus conventional craniotomy in the treatment of acute intracerebral hemorrhage. BMC Neruol 2011;11:76

7. Kuo LT, Chen CM, Li CH, et al. Early endoscope-assisted hematoma evacuation in patients with supratentorial intracerebral hemorrhage: case selection, surgical technique, and long-term results. Neurosurg Focus 2011;30:E9

8. Barlas O, Karadereler S, Bahar S, et al. Image-guided keyhole evacu- ation of spontaneous supratentorial intracerebral hemorrhage. Minim Invasive Neurosurg 2009;52:62-68

9. Morgan T, Zuccarello M, Narayan R, et al. Preliminary findings of the minimally-invasive surgery plus rtPA for intracerebral hemorrhage evacuation (MISTIE) clinical trial. Acta Neurochir Suppl 2008;105:147-51

10. Dey M, Stadnik A, Awad IA. Spontaneous intracerebral and intraventricular hemorrhage: advances in minimally invasive surgery and thrombolytic evacuation, and lessons learned in recent trials. Neurosurgery 2014;74(suppl 1):S142-50

11. Kyriakou Y, Struffert T, Dorfler A, et al. Basic principles of flat detector computed tomography (FD-CT). Der Radiologe 2009;49: 811-19

12. Freundt MI, Ritter M, Al-Zghloul M, et al. Laser-guided cervical selective nerve root block with the Dyna-CT: initial experience of three-dimensional puncture planning with an ex-vivo model. PloS One 2013;8:e69311

13. Ritter M, Rassweiler MC, Hacker A, et al. Laser-guided percutaneous kidney access with the Uro Dyna-CT: first experience of three-dimensional puncture planning with an ex vivo model. World J Urol 2013;31:1147-51

14. Fujisawa M, Yamashita S, Katagi R. Usefulness of intraoperative computed tomography for the evacuation of lobar hemorrhage. Acta Neurochir Suppl 2013;118:175-79

15. Bhattathiri PS, Gregson B, Prasad KS, et al. Intraventricular hemorrhage and hydrocephalus after spontaneous intracerebral hemorrhage: results from the STICH trial. Acta Neurochir Suppl 2006;96:65-68

16. Hanley DF. Intraventricular hemorrhage: severity factor and treatment target in spontaneous intracerebral hemorrhage. Stroke 2009;40:1533-38

17. Rossitti S. Spontaneous intracerebral and intraventricular hemorrhage. Hemostasis by transarterial glue embolization. Clin Neuroradiol 2010;20:131-34

18. Marquardt G, Wolff R, Janzen RW, et al. Basal ganglia haematomas in non-comatose patients: subacute stereotactic aspiration improves long-term outcome in comparison to purely medical treatment. Neurosurg Rev 2005;28:64-69

19. Tang ZP, Shi YH, Yin XP, et al. Modifying the details of aspiration operation may contribute to the improvement of prognosis of patients with ICH. Turk Neurosurg 2012;22:13-20 